\title{
Quantification of Blood Velocity with 4D Digital Subtraction Angiography Using the Shifted Least-Squares Method
}

\author{
(D) Y. Wu, (D) G. Shaughnessy, (D) C.A. Hoffman, (DE.L. Oberstar, (D). Schafer, (D)T. Schubert, (D).L. Ruedinger, (D) B.J. Davis, \\ (iDC.A. Mistretta, (DC.M. Strother, and (D).A. Speidel
}

\begin{abstract}
BACKGROUND AND PURPOSE: 4D-DSA provides time-resolved 3D-DSA volumes with high temporal and spatial resolutions. The purpose of this study is to investigate a shifted least squares method to estimate the blood velocity from the 4D DSA images. Quantitative validation was performed using a flow phantom with an ultrasonic flow probe as ground truth. Quantification of blood velocity in human internal carotid arteries was compared with measurements generated from 3D phase-contrast MR imaging.
\end{abstract}

MATERIALS AND METHODS: The centerlines of selected vascular segments and the time concentration curves of each voxel along the centerlines were determined from the 4D-DSA dataset. The temporal shift required to achieve a minimum difference between any point and other points along the centerline of a segment was calculated. The temporal shift as a function of centerline point position was fit to a straight line to generate the velocity. The proposed shifted least-squares method was first validated using a flow phantom study. Blood velocities were also estimated in the 14 ICAs of human subjects who had both 4D-DSA and phase-contrast MR imaging studies. Linear regression and correlation analysis were performed on both the phantom study and clinical study, respectively.

RESULTS: Mean velocities of the flow phantom calculated from 4D-DSA matched very well with ultrasonic flow probe measurements with $11 \%$ relative root mean square error. Mean blood velocities of ICAs calculated from 4D-DSA correlated well with phase-contrast MR imaging measurements with Pearson correlation coefficient $r=0.835$.

CONCLUSIONS: The availability of 4D-DSA provides the opportunity to use the shifted least-squares method to estimate velocity in vessels within a 3D volume.

ABBREVIATIONS: $\mathrm{PC}=$ phase-contrast; $\mathrm{SBR}=$ sideband ratio; $\mathrm{TCC}=$ time concentration curve; $\mathrm{VIPR}=$ vastly undersampled isotropic projection reconstruction

2 D digital subtraction angiography and a 3D rotational angiography acquisition followed by $3 \mathrm{D}$-DSA reconstruction are the standards for vascular morphology assessment. There is an increasing demand for hemodynamic information, including blood flow rate and velocity for diagnosis, treatment planning, and evaluation. ${ }^{1-3}$ Many algorithms have been proposed to estimate blood

Received February 6, 2018; accepted after revision June 11.

From the Departments of Medical Physics (Y.W., G.S., C.A.H., C.A.M., M.A.S.), Biomedical Engineering (E.L.O., K.L.R., B.J.D.), Radiology (C.A.M., C.M.S., T.S.), and Medicine (M.A.S.), University of Wisconsin, Madison, Wisconsin; Siemens Healthineers (S.S.), USA; and Department of Radiology and Nuclear Medicine (T.S.), Basel University Hospital, Basel, Switzerland.

This work was supported by the National Institutes of Health Center for Scientific Review 2R01HL116567 and Siemens Healthineers.

The concepts and information presented in this article are based on research and are not commercially available.

Please address correspondence to Yijing Wu, MD, Department of Medical Physics, University of Wisconsin, Madison, 1125 WIMR, 1111 Highland Ave, Madison, WI 53705; e-mail: yijingwu@wisc.edu

- Indicates open access to non-subscribers at www.ajnr.org

http://dx.doi.org/10.3174/ajnr.A5793 flow in arteries from 2D-DSA; these are divided into 2 major classes: bolus-tracking and computational methods. ${ }^{4-11} \mathrm{~A}$ thorough review of these techniques can be found in Shpilfoygel et al. ${ }^{12}$ However, 2D-DSA based methods are challenged by vessel overlap and vessels being aligned with the primary $\mathrm{x}$-ray beam direction.

Recently, a new reconstruction method ${ }^{13-16}$ has been applied to a single $3 \mathrm{D}$ rotational angiography acquisition to generate time-resolved 3D-DSA at frame rates up to 30 frames/second (4D DSA). The availability of the geometric and temporal data in a 4D-DSA reconstruction provides the opportunity to estimate velocity and flow more accurately than has previously been possible with 2D DSA. In this article, a shifted least-squares based technique was used with the 4D-DSA data to estimate blood velocity. The proposed algorithm was first validated using flow phantom studies, in which the velocity could be documented using an ultrasonic flow probe. Blood velocity was also determined in $4 \mathrm{D}$ DSA datasets from 14 ICAs of volunteers who also underwent 3D fast phase-contrast with vastly undersampled isotropic projection reconstruction (PC VIPR) studies. ${ }^{17-19}$ 


\section{MATERIALS AND METHODS}

\section{D DSA Acquisition and Data Preparation}

Noncontrast (mask) and contrast-enhanced (fill) rotational acquisitions were performed using a conventional flat panel detector angiographic system (Artis zee; Siemens, Erlangen, Germany) with the following settings: $70 \mathrm{kV}$ (peak), $0.36 \mu \mathrm{Gy} /$ frame, 304 images, $260^{\circ}$ arc. Contrast injection (iohexol, Omnipaque 300; GE Healthcare, Piscataway, New Jersey) into the subject was started at the beginning of the fill run $(3 \mathrm{~mL} /$ second, 7 -second injection duration). The rotational acquisition protocol yields $\mathrm{x}-$ ray projection images, which are used to automatically reconstruct both 3D-DSA and 4D-DSA with an isotropic spatial resolution of $0.46 \times 0.46 \times 0.46 \mathrm{~mm}^{3}$.

The centerline of each vascular segment was determined from the static $3 \mathrm{D}$ volume using an efficient $3 \mathrm{D}$ parallel-thinning algorithm. ${ }^{20}$ The contrast waveform map, which is the time concentration curves (TCCs) along the centerline path (curvilinear length $z$ ), was extracted from the 4D-DSA and stored for each vascular segment for velocity calculation.

\section{Shifted Least-Squares Algorithm}

In an arterial contrast injection, there is a temporal oscillation in iodine concentration that arises from the mixing of contrast medium, which is injected at a fixed rate, and nonopacified blood, which flows at a variable rate driven by the cardiac cycle. This temporal variation in contrast, referred to as pulsatility, appears at points downstream of the injection with a time delay. The time delay is related to the distance downstream and the blood velocity. Therefore, velocity can be estimated by measuring the distance along the vessel centerline and the time delay.

For any 2 points, $i$ and $j$, along the centerline, the shifted leastsquares difference between their TCCs, $c_{\mathrm{i}}(t)$ and $c_{\mathfrak{j}}(t)$, can be calculated as

$$
\varepsilon\left(\tau_{\mathrm{ij}}\right)=\frac{1}{T} \sqrt{\sum_{\mathrm{t}=1}^{T}\left[c_{\mathrm{i}}\left(t-\tau_{\mathrm{ij}}\right)-c_{\mathrm{j}}(t)\right]^{2}} .
$$

The value $\tau_{\mathrm{ij}}^{0}, \tau_{\mathrm{ij}}^{0} \in[0, T]$ that minimizes the shifted least-squares difference, $\varepsilon\left(\tau_{\mathrm{ij}}\right)$, is regarded as the time of bolus transport between these 2 points. The spatial distance $z$ between the 2 points is calculated from the 3D path length along the vessel centerline. The time-shift $\tau^{0}$ as a function of the spatial distance $z$ can then be fit to a linear relation:

$$
\tau^{0}=\alpha \times z+b
$$

where the slope $a$ is the inverse of the velocity $v$.

\section{Optimizing Waveform Selection}

It has been found that the shifted least-squares algorithm provides the most reliable velocity calculation in vessel segments where pulsatility is strong and consistent. To automatically select the waveform regions with strong and consistent pulsatility, we generated a sideband ratio (SBR) map. ${ }^{21}$

For each point in the waveform map, a short-time Fourier transform was applied to generate the local power spectrum $P S_{\mathrm{fn}}$, where $f_{\mathrm{n}}$ is the $n^{\text {th }}$ characteristic frequency. The SBR at this point can be calculated as

$$
S B R=\frac{\left|P S_{\mathrm{f}_{0}}\right|^{2}}{\frac{1}{N} \sum\left|P S_{\mathrm{f}_{\mathrm{i}}}\right|^{2}},
$$

where the denominator summation includes the neighboring frequency points and excludes the fundamental frequency $f_{0}$. From this SBR map, a low-pass filter was applied and a threshold of one-fourth of the median SBR was enforced to generate a mask. This mask excludes low pulsatile signals from consideration in the velocity calculation.

\section{Phantom Study}

To validate the proposed method for velocity estimation, we used a flow phantom containing a loop of plastic tubing to perform 4D-DSA studies. Pulsatile water flow with different mean flow rates was generated using a perfusion pump with a controllable flow setting (S3; Storkcert, Freiburg, Germany). A 5F catheter was introduced into the tubing for injection of iodinated contrast medium (Omnipaque 300 diluted with 25\% water) with a power injector. 4D-DSA-derived flow was compared with flow measurements made with an ultrasonic flow probe (400-Series MultiChannel Flowmeter; Transonic, Ithaca, New York) attached to the tubing 5 inches away from the catheter tip.

Rotational projection images were acquired using the mask and iodine-filled scans with 12 -second acquisition over a $260^{\circ}$ arc. The frame rate was 30 frames per second. The 4D-DSA volume data were reconstructed using the vendor's prototype software (Siemens Healthineers, Forchheim, Germany).

A time-resolved flow profile was recorded during each fill scan using the ultrasonic probe. The reference flow measurement was taken as the average of the flow profile over the same time window that was used for 4D-DSA-derived flow.

Five different flow settings were selected to provide average flow rates ranging from 285 to $1140 \mathrm{~mL} / \mathrm{min}$ (corresponding to the mean velocity of $15-60 \mathrm{~cm} / \mathrm{s}$ for the tubing with a $0.64-\mathrm{cm}$ diameter). Three 4D-DSA scans and reference flow measurements were made for each flow setting.

\section{Clinical Study}

Nine subjects who had both DSA and PC VIPR examinations were selected from a data base generated under University of Wisconsin review board approval. Because 5 of 9 subjects had bilateral studies, a total of 14 ICAs were available for evaluation. Scanning parameters for postcontrast PC VIPR were the following: $\mathrm{FOV}=$ $22 \times 22 \times 22 \mathrm{~cm}^{2}, \mathrm{TR} / \mathrm{TE}=12.5 / 4.8 \mathrm{~ms}$, velocity-encoded gradient-echo imaging $=80 \sim 100 \mathrm{~cm} / \mathrm{s}$, bandwidth $=83.3 \mathrm{kHz}$, readout matrix $=320$ points per projections, spatial resolution for the complex difference image $=0.68 \times 0.68 \times 0.68 \mathrm{~mm}^{3}$. The complex difference images from the PC VIPR scan were segmented for vessel content using Materialise Mimics (Materialise NV, Leuven, Belgium). The segmented MR imaging data were then registered to the 3D-DSA using a correlated point registration with manual point placement, followed by an affine rigid registration. Centerline coordinates of the ICAs generated from the 3D-DSA volumes were used for velocity estimation. An automated workflow for the PC VIPR analysis was developed in Matlab (MathWorks, Natick, Massachusetts). This software performed cross-sectional plane placement for every voxel along the 

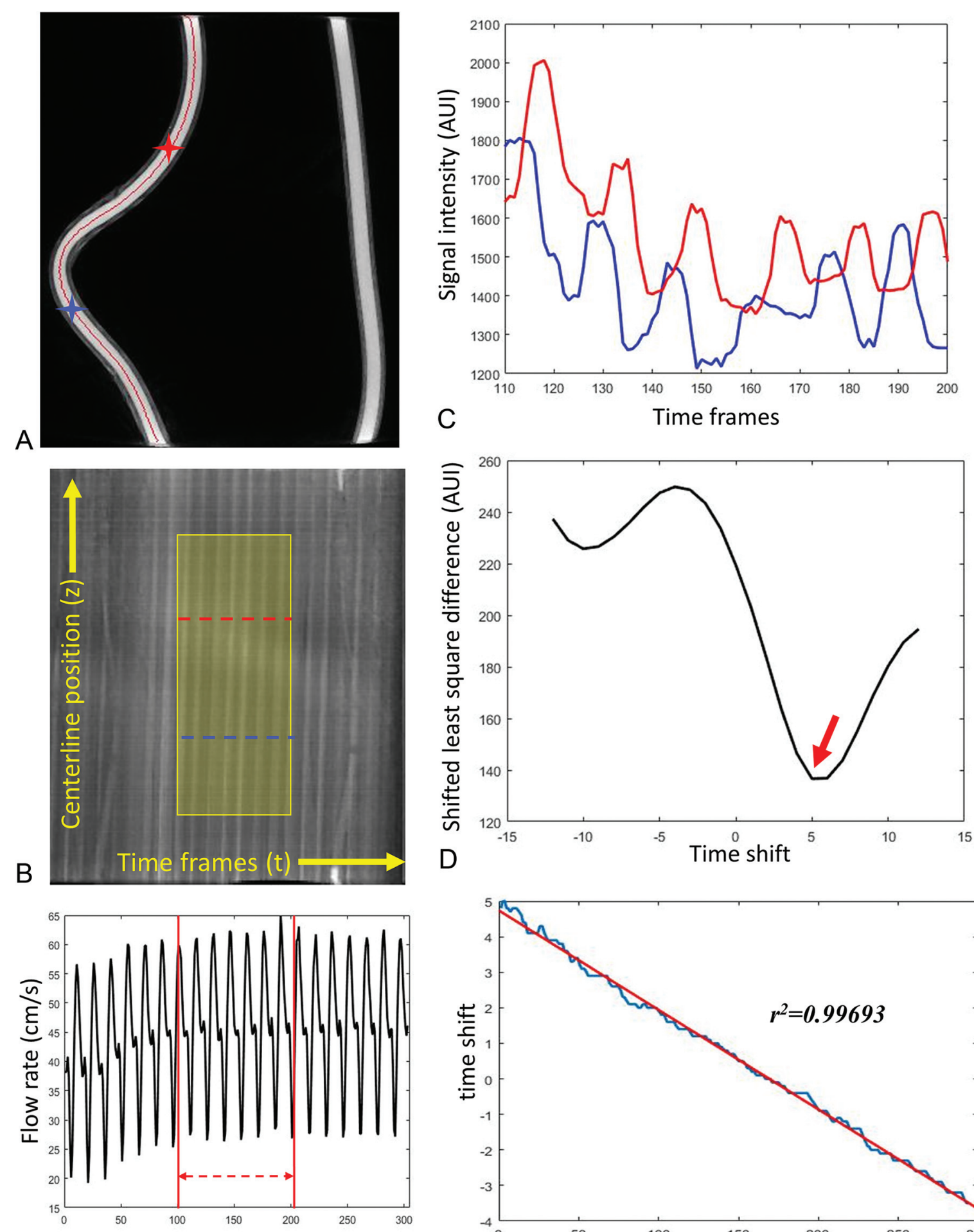

$E$

Time frames

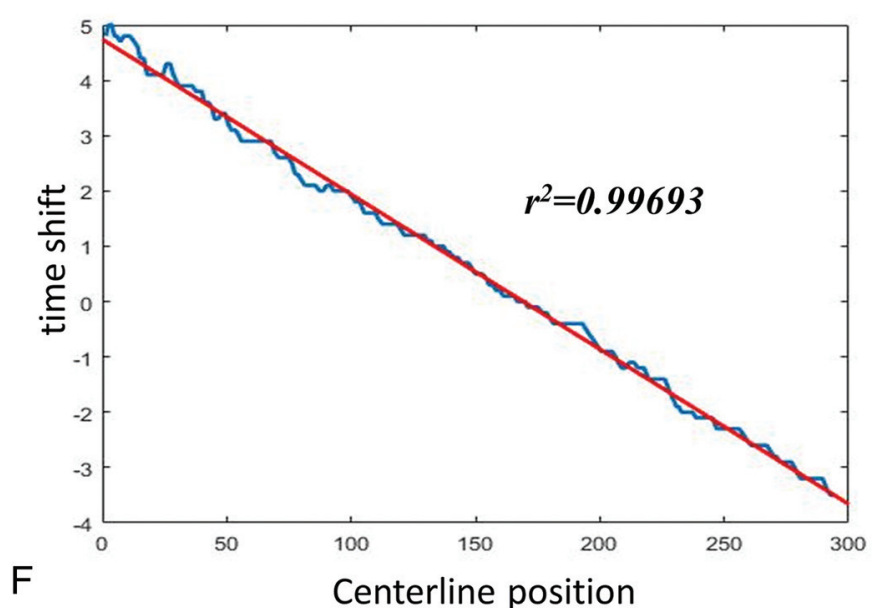

FIG 1. A representative phantom study demonstrates the flow calculation from 4D-DSA. A, MIP image of the reconstructed phantom with centerline (red) overlaid. $B$, The contrast waveform map of the voxels along the centerline. The horizontal direction is the timeframe in the $4 D-D S A$ scan, and the vertical direction is the position along the vessel centerline. Each point in the map $M(t, z)$ represents the signal intensity of the voxel at distance $z$ along the centerline and at time frame $t$. The highlighted area is the optimized waveform region where the pulsatility is strong and consistent. TCCs of 2 selected voxels along the centerline (marked as blue and red stars on $A$ and blue and red curves on $B$ ) are shown in C.D. The least-squares differences of these 2 signals as a function of the time-shift, in which the minimal appears at $\tau^{0}=5$ shown as red arrow on $\mathrm{D}$ is considered as the time of bolus transport from the blue voxel to the red voxel. The time-shift as a function of the centerline position was fit to a linear relation $(F)$, where the slope is the inverse of the velocity. $E$, The flow profile recorded from the flow probe (downsampled to $1 / 30$ second to correspond to the 4D-DSA timeframes). Flow measurement is the average taken between the red lines, which correspond to the optimized window shown in B. AUI indicates arbitrary unit of intensity. 
3D centerline path, segmentation of the vessel boundaries in each plane, and averaging of the normal velocities for all points inside the segmented boundaries. The reported velocity value for a vessel was then the average result over all the centerline points that were present in both the PC VIPR and 3D-DSA volumes.

\section{Statistical Analysis}

The validation process was performed using linear regression fit between flow probe $\left(V_{\mathrm{fp}}\right)$ and $V_{\mathrm{dsa}}$ (calculated using 4D-DSA) for the phantom study. Correlation analysis and Bland-Altman plots were used to compare the velocity measurements using PC VIPR

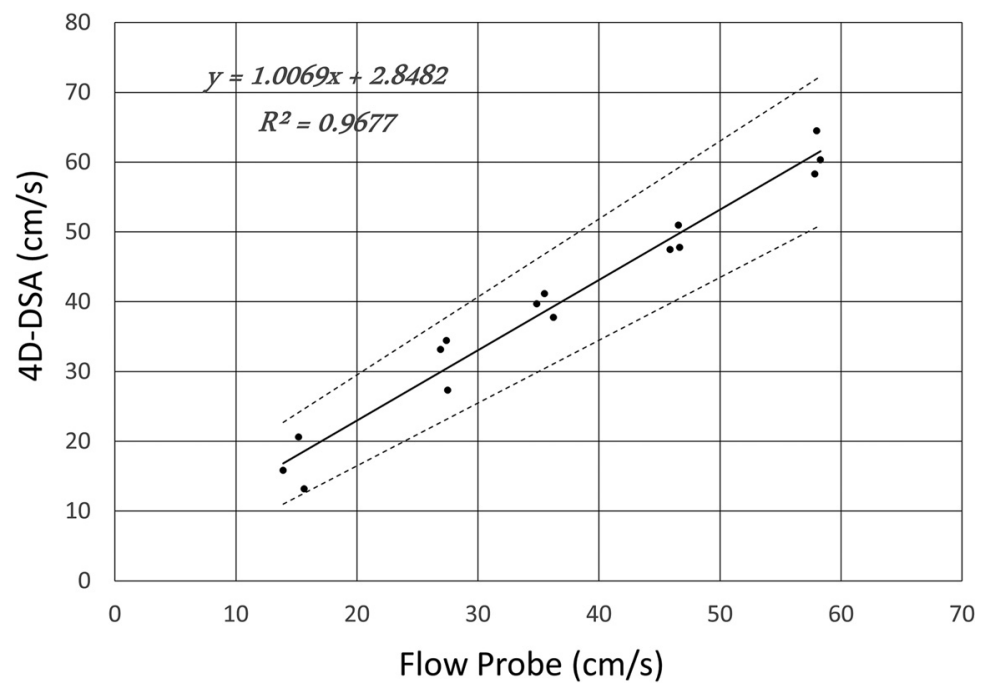

FIG 2. Linear regression between the flow probe measurements and the 4D-DSA calculations from 15 phantom studies. Evaluations have been obtained with significance levels $P=4.521 \mathrm{E}-11 \mathrm{for}$ the slope and $P=0.18$ for the intercept. versus 4D-DSA for the clinical study. The Pearson $r$ was used for correlation analysis, and a $P$ value $<.05$ for the correlation coefficient was considered statistically significant.

\section{RESULTS}

\section{Phantom Study}

Figure 1 shows sample data from the phantom study. First, the centerline was generated from the static 3D volume (Fig 1A). Figure $1 B$ is the contrast waveform map (TCCs along the centerline path) of the selected centerline. Each point in the map $M(t, z)$ represents the signal intensity of the voxel at distance $z$ along the centerline and at timeframe $t\left[C_{z}(t)\right]$. The intensity variations along the vertical axis reflect the concentration of iodine contrast medium versus the distance from the injection site for a given time point. Variations along the horizontal axis reflect variations in concentration versus time for a given point along the centerline. The slope of the intensity ridges reflects the blood velocity. In the displayed format, higher blood velocities produce ridges that are closer to vertical, whereas lower blood velocities produce ridges closer to horizontal. The highlighted region is the optimized waveform region for the flow calculation determined using the SBR method. TCCs of 2 selected voxels along the centerline (marked as blue and red stars on Fig $1 A$ and blue and red dashed lines on
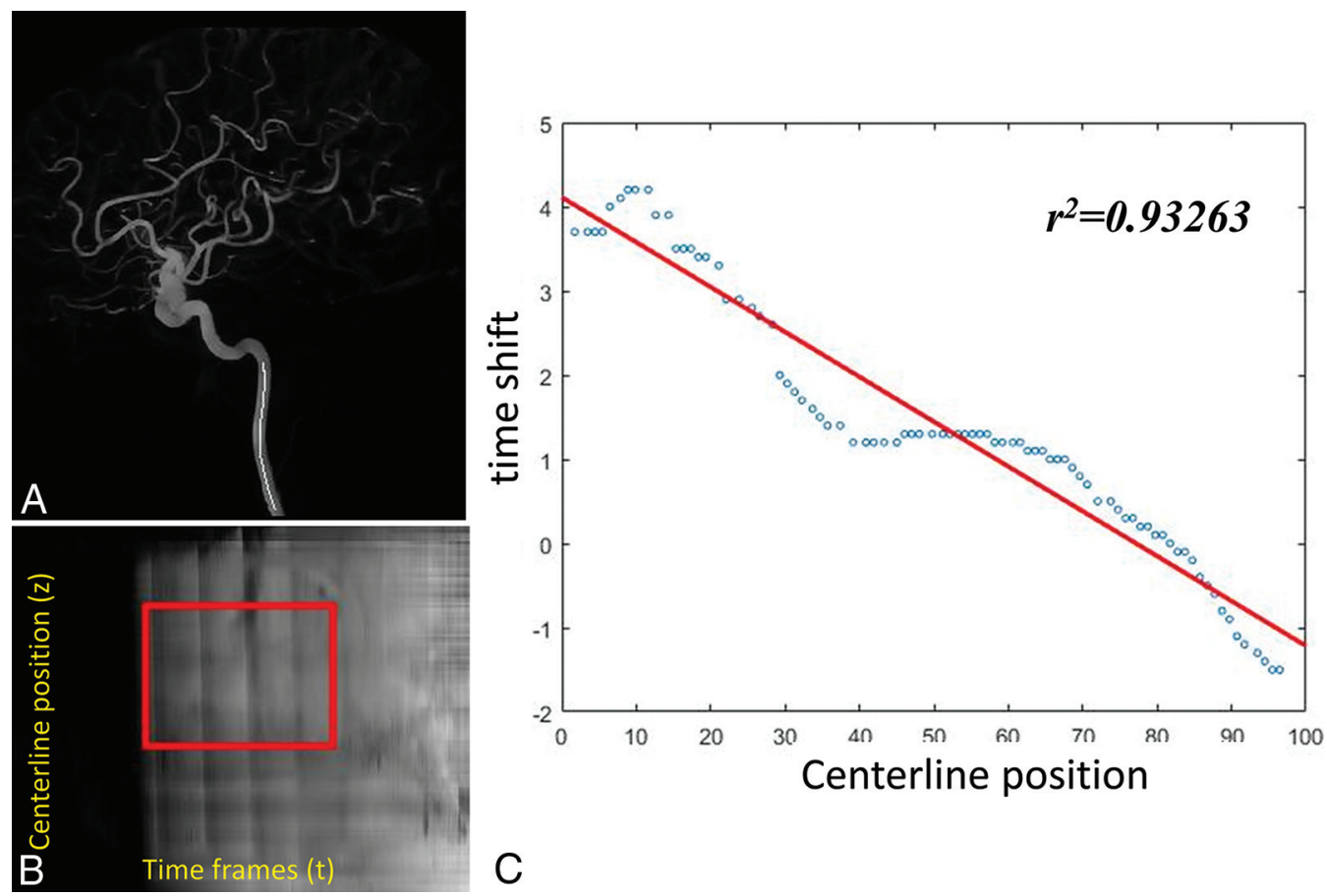

FIG 3. A representative ICA study. A, The centerline positions of the ICA. $B$, The contrast waveform map of the voxels along the centerline. The highlighted area is the optimized waveform region for the flow calculation. $C$, The time-shift as a function of the centerline position was fit to a linear relation in which the slope is the inverse of the velocity. 


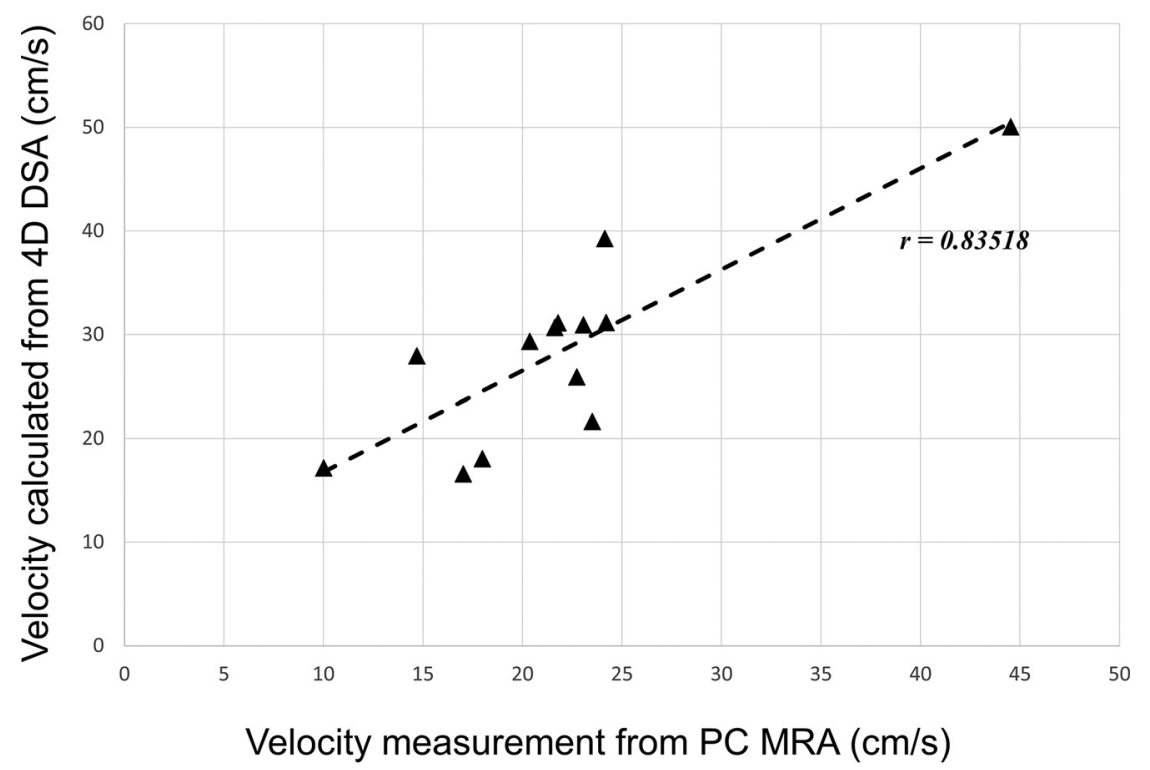

FIG 4. Correlation analysis between velocity estimated from 4D-DSA and measured using PC VIPR. The significance level is $P=.0002$.

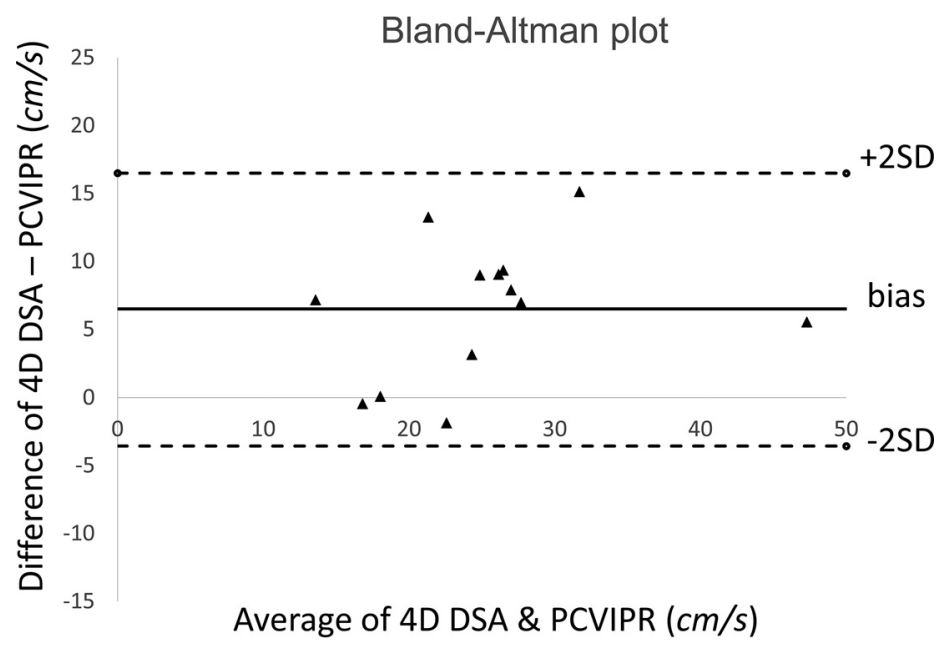

FIG 5. Bland-Altman analysis of 4D-DSA compared with PC VIPR.

Fig $1 B$ ) are shown in Fig $1 C$. Figure $1 D$ shows the least-squares differences of these 2 signals as a function of the time-shift, where the minimal appears at $\tau^{0}=5$ is considered as the time of bolus transport from the blue voxel to the red voxel. Figure $1 F$ demonstrates the linear fitting between the time-shift of each voxel along the centerline and its corresponding centerline position. Figure $1 E$ is the flow profile recorded from the flow probe (downsampled to $1 / 30$ second to correspond to the $4 \mathrm{D}$-DSA timeframes). Flow measurement was taken as an average over the same temporal window used in the 4D-DSA flow calculation.

The linear regression fit between the flow probe measurement and the 4D-DSA calculation is shown in Fig 2. The linear regression equation is as follows: $V_{\mathrm{dsa}}=1.0069 V_{\mathrm{fp}}+2.8482$, where $V_{\mathrm{dsa}}$ is the velocity calculated from $4 \mathrm{D}$-DSA and $V_{\mathrm{fp}}$ is the measurement using the flow probe. The determination coefficient $R^{2}$ of the fit was 0.9677 . The $95 \%$ confidence interval slope was $0.8966-$ 1.1172 and the $95 \%$ CI intercept was $-1.4917-7.1881$. These evaluations have been obtained with significance levels of $P=$
$4.521 \mathrm{E}-11$ and $P=0.18$, respectively. The relative root mean square error (normalized by mean velocity) was $11.3 \%$.

\section{Clinical Study}

The average ICA flow rates were calculated from 13 of the 14 ICAs that had 4D-DSA examinations. One subject was excluded due to the poor pulsatility in the TCCs. Figure 3 shows an example of the ICA studies. Average ICA flow rates were also measured with the PC VIPR studies. Correlation between 2 measurements using 4D-DSA and PC VIPR is shown in Fig 4. Average ICA flow rates calculated using 4D-DSA and measured using PC VIPR are well-correlated, with the Pearson $r=0.835$ and a significance

level $P=.0002$. Figure 5 is the Bland-Altman analysis of $4 \mathrm{D}$-DSA compared with PC VIPR, which shows all points within 2 SDs, with a bias of $6.5 \mathrm{~cm} / \mathrm{s}$ and 2-SD limits of agreement of $-3.56 \mathrm{~cm} / \mathrm{s}$ and $16.5 \mathrm{~cm} / \mathrm{s}$.

\section{DISCUSSION}

The shifted least-squares algorithm is a simple-but-robust method to calculate flow rates by finding the bolus transport time between any 2 points along the centerline and performing linear regression with the corresponding positions. The combination of the spatial and temporal data available in the 4D-DSA reconstruction provides the opportunity to apply this technique to measurement of blood flow in routine 4D-DSA acquisitions. The flow phantom study demonstrated that by using this method, we were able to calculate the flow rates with $11 \%$ relative root mean square error compared with ultrasonic flow probe measurements. Results from the 13 human ICAs showed that the calculated velocities from 4D-DSA studies correlated well with the measurements 

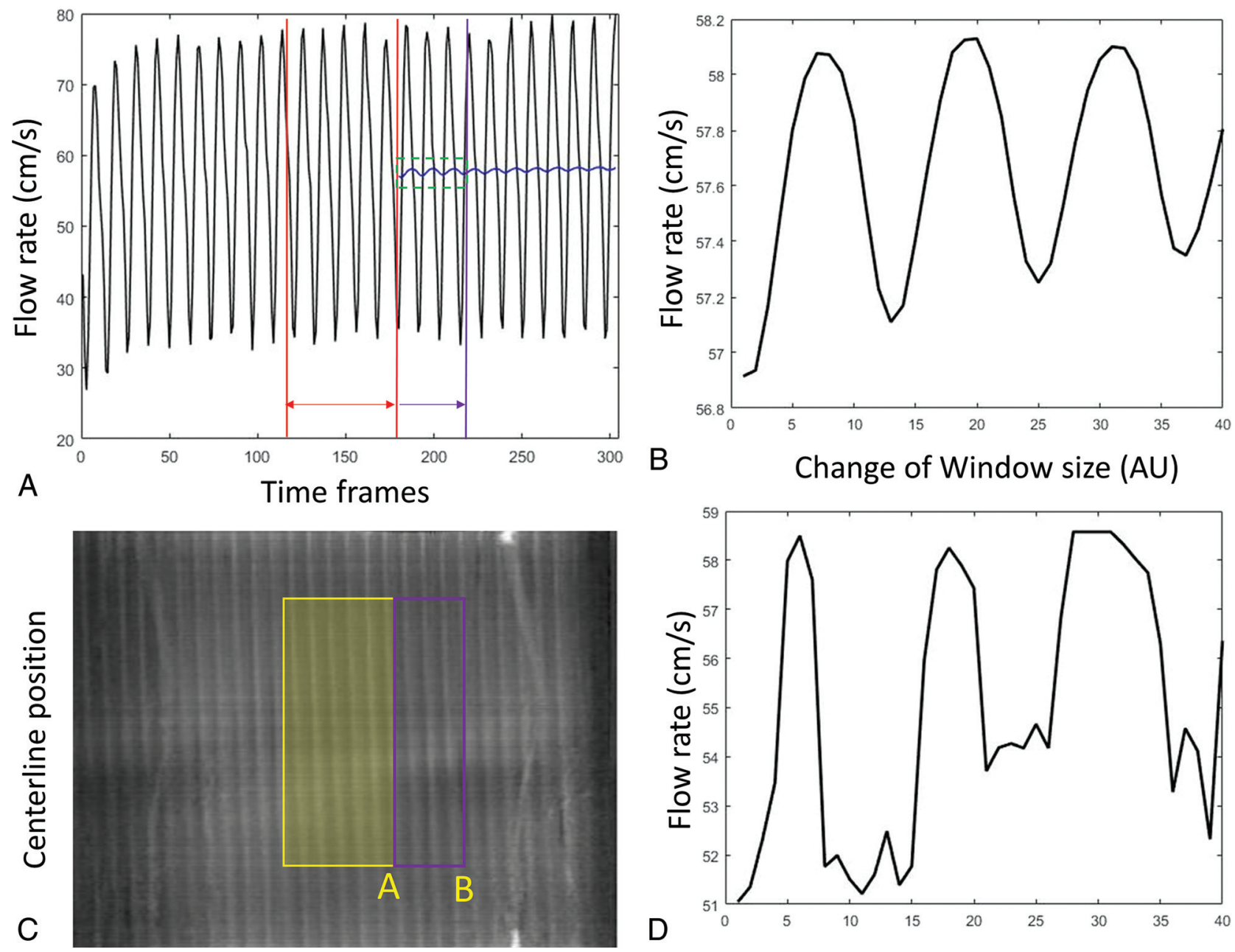

FIG 6. Fluctuation of the velocity estimation with a different average range. $A$, The flow profile recorded from the flow probe. Red lines define the starting average window. This window was gradually expanded to the purple line. The corresponding mean velocity was shown as the blue curve. A zoom-in of the green window is shown in $B$. Similarly, velocity calculations have been performed by gradually expanding the window coverage from yellow to purple as shown in the waveform map $(C)$. Estimated velocity varies $(D)$ as the window edge slides from position $A$ toward B. AU indicates arbitrary units.

using the 3D phase-contrast MR imaging method. We believe that the reason for the higher velocities in the $4 \mathrm{D}$-DSA studies compared with the PC VIPR measurements $(6.5 \mathrm{~cm} / \mathrm{s}$ bias $)$ is the increment in flow caused by the intra-arterial power injection of contrast medium.

The proposed method calculates the mean flow rate within a selected window defined by the SBR map. This truncated average of pulsatile flow varies depending on the window size and location. This variation can be demonstrated in Fig $6 A$ and $B$, where the average of the flow profile was performed with a gradually expanding average window. The truncated average oscillates depending on where the truncation window ends and eventually converges to the mean signal as the window expands. Similar oscillation has been observed in the flow calculation using 4D-DSA. Figure $6 D$ shows the estimated average velocity changes as the right side of the window gradually expands as shown in Fig 6C. The current algorithm automatically selects a window purely on the basis of the SBR map so that the selected window range may fall anywhere between 4 and 6 pulsatile cycles. Further criteria could be included to trim the window to an integer number of pulsatile cycles to minimize this error.
The proposed algorithm worked best when there was good and consistent pulsatility in the 4D-DSA TCCs. With each cardiac cycle, as an intra-arterial contrast bolus is injected, there is an oscillation in the ratio of contrast medium to nonopacified blood. This pulsatility can be identified and extracted from the contrast waveform map by thresholding the SBR map. For vessels more distal to the contrast injection site where the pulsatility can be low, injection protocols should be modified to enhance the pulsatility strength or contrast kinetics. The present work focused on the evaluation of blood velocity within the ICAs. Investigation of more downstream segments, including the MCAs and basilar arteries, should be performed in the future.

\section{CONCLUSIONS}

4D DSA is a novel technique that provides both the geometric and temporal information required to measure blood flow velocity and flow. In this study, calculated velocity values from 4D-DSA TCCs correlated well with human ICA measurements derived from phase-contrast MR imaging and flow phantom measurements using an ultrasonic flow probe. 
Disclosures: Gabe Shaughnessy—RELATED: Grant: National Institutes of Health.* Erick L. Oberstar-RELATED: Grant: University of Wisconsin, Madison, Comments: National Institutes of Health Grant R01HL116567*. Sebastian Schafer-UNRELATED: Employment: Siemens Healthineers, Comments: full-time employee. Michael A. Speidel—RELATED: Grant: Siemens Healthineers, Comments: Partial support was received from a research grant*; UNRELATED: Patents (Planned, Pending or Issued): Wisconsin Alumni Research Foundation, Comments: I am a coinventor on a Wisconsin Alumni Research Foundation patent (pending) on resolving artifacts in 4D angiographic data. Money of the patents was paid to Wisconsin Alumni Research Foundation. Charles A. Mistretta-RELATED: Grant: National Institute of Biomedical Imaging and Bioengineering, Comments: Strother/Mistretta R01 on 4DDSA*; UNRELATED: Patents (Planned, Pending or Issued): Wisconsin Alumni Research Foundation. Charles M. Strother-RELATED: Grant: University of Wisconsin School of Medicine and Public Health*; UNRELATED: Patents (Planned, Pending or Issued): Wisconsin Alumni Research Foundation, Comments: I have a pending IP for flow quantification; the IP is assigned to the Wisconsin Alumni Research Foundation. *Money paid to the institution.

\section{REFERENCES}

1. Murayama Y, Usami S, Abe T, et al. Transvenous Doppler guidewire sonographic monitoring during treatment of a complex vertebral arteriovenous fistula associated with neurofibromatosis type 1. Neuroradiology 1999;41:328-33 CrossRef Medline

2. Mast H, Mohr J, Thompson J, et al. Transcranial Doppler ultrasonography in cerebral arteriovenous malformations: diagnostic sensitivity and association of flow velocity with spontaneous hemorrhage and focal neurological deficit. Stroke 1995;26:1024-27 CrossRef Medline

3. Turski P, Levine R, Turnipseed W, et al. MR angiography flow analysis: neurovascular applications. Magn Reson Imaging Clin $\mathrm{N}$ Am 1995;3:541-55 Medline

4. Rosen L, Silverman NR, Videodensitometric measurements of blood flow using crosscorrelation techniques. Radiology 1973;109: 305-10 CrossRef Medline

5. Silverman NR, Rosen L. Arterial blood flow measurement: assessment of velocity estimation methods. Invest Radiol 1977:12:319-24 CrossRef Medline

6. Seifalian AM, Hawkes DJ, Colchester AC, et al. A new algorithm for deriving pulsatile blood flow waveforms tested using simulated dynamic angiographic data, Neuroradiology 1989;31:263-69 Medline

7. Hoffmann KR, Doi K, Fencil LE. Determination of instantaneous and average blood flow rates from digital angiograms of vessel phantoms using distance-density curves. Invest Radiol 1991;26: 207-12 Medline

8. Imbert B, Meunier J, Mongrain R, et al. Stenosis parameter assessment from contrast medium tracking in cineangiography with an optical flow method. Proc SPIE Int Soc Opt Eng 1997;13034:631-40 CrossRef
9. Pereira VM, Bonnefous O, Ouared R, et al. A DSA-based method using contrast-motion estimation for the assessment of the intraaneurysmal flow changes induced by flow-diverter stents. AJNR Am J Neuroradiol 2013;34:808-15 CrossRef Medline

10. Rhode KS, Lambrou T, Hawkes DJ, et al. Novel approaches to the measurement of arterial blood flow from dynamic digital X-ray images. IEEE Trans Med Imaging 2005;24:500-13 CrossRef Medline

11. Pereira VM, Ouared R, Brina O, et al. Quantification of internal carotid artery flow with digital subtraction angiography: validation of an optical flow approach with Doppler ultrasound. AJNR Am J Neuroradiol 2014;35:156-63 CrossRef Medline

12. Shpilfoygel SD, Close RA, Valentino DJ, et al. X-ray videodensitometric methods for blood flow and velocity measurement: a critical review of literature. Med Phys 2000;27:2008-23 CrossRef Medline

13. Mistretta CA, Oberstar EL, Davis JB, et al. 4D DSA and 4D fluoroscopy: preliminary implementation. Proc SPIE Int Soc Opt Eng 2010;7622 CrossRef

14. Strother CM, Bender F, Deuerling-Zheng Y, et. al. Parametric color coding of digital subtraction angiography. AJNR Am J Neuroradiol 2010;31:919-24 CrossRef Medline

15. Benndorf $G$. Color-coded digital subtraction angiography: the end of a monochromatic era? AJNR Am J Neuroradiol 2010;31:925-27 CrossRef Medline

16. Davis B, Royalty K, Kowarschik M, et al. 4D digital subtraction angiography: implementation and demonstration of feasibility. AJNR Am J Neuroradiol 2013;34:1914-21 CrossRef Medline

17. Gu T, Korosec FR, Block W, et al. PC VIPR: a high-speed 3D phasecontrast method for flow quantification and high-resolution angiography. AJNR Am J Neuroradiol 2005;26:743-49 Medline

18. Chang W, Landgraf B, Johnson KM, et al. Velocity measurements in the middle cerebral arteries of healthy volunteers using 3D radial phase-contrast HYPRFlow: comparison with transcranial Doppler sonography and $2 \mathrm{D}$ phase-contrast MR imaging. AJNR Am J Neuroradiol 2011;32:54-59 CrossRef Medline

19. Schneiders JJ, Ferns SP, van Ooij P, et al. Comparison of phasecontrast MR imaging and endovascular sonography for intracranial blood flow velocity measurements. AJNR Am J Neuroradiol 2012;33:1786-90 CrossRef Medline

20. Lee TC, Kashyap RL, Chu CN. Building skeleton models via 3-D medial surface axis thinning algorithms. Graphical Models and Image Processing 1994;56:462-78 CrossRef

21. Shaughnessy G, Hoffman C, Schafer S, et al. Quantitative flow and velocity measurements of pulsatile blood flow with 4D DSA. Proc SPIE 10132, Medical Imaging 2017: Physics of Medical Imaging, 101325R (19 April 2017); doi: 10.1117/12.2254143 CrossRef 